\title{
Swift Heavy Ion Irradiation of Nickel Nanoparticles
}

D. J. Sprouster ${ }^{1}$, R. Giulian ${ }^{1}$, D. J. Llewellyn ${ }^{1,3}$, P. Kluth ${ }^{1}$, L. L. Araujo ${ }^{1}$, J. D. Fitzgerald $^{2}$, M. C. Ridgway ${ }^{1}$

${ }^{1}$ Department of Electronic Materials Engineering, Research School of Physics and Engineering, Australian National University, Canberra, ACT 0200, Australia

${ }^{2}$ Research School of Earth Sciences, Australian National University, Canberra, 0200, Australia

${ }^{3}$ Centre for Advanced Microscopy, Australian National University, Canberra, ACT 0200, Australia

Metallic nanoparticles (NPs) embedded in a silica matrix have potential applications in ultrahigh-density magnetic recording and in photonic devices ${ }^{1}$. Both the optical and magnetic properties are highly dependent on the size and shape of the NP ensemble ${ }^{2,3}$. For non-spherical shapes, NP orientation is also important and permits the optimization of both the optical and magnetic properties ${ }^{4}$. The ability to control the aforementioned properties is thus essential for the efficient integration of NPs in advanced devices. Swift heavy ion irradiation (SHII) is a novel means of modifying embedded NPs with a spherical to rod-like shape transformation ${ }^{3,5,6}$. In this work, cross-sectional transmission electron microscopy (XTEM) is used in conjunction with small-angle X-ray scattering (SAXS) to characterize the effects of SHII on Ni NPs embedded in a $\mathrm{SiO}_{2}$ matrix. We show that elongation of the once spherical NPs is readily achievable and further demonstrate the effectiveness of SHII as a means of tailoring the size, shape and orientation of metallic nanoparticles.

Ni NPs were synthesized in a $2 \mu \mathrm{m} \mathrm{SiO}_{2}$ layer grown on a $520 \mu \mathrm{m} \mathrm{Si} \mathrm{(100)} \mathrm{wafer} \mathrm{by} \mathrm{ion}$ implantation and thermal annealing. The NPs were then irradiated with $185 \mathrm{MeV} \mathrm{Au}$ ions in a wide fluence range $\left(10^{11}-10^{14} / \mathrm{cm}^{2}\right)$ at room temperature. XTEM samples were prepared by bonding a NP sample between two microscope glass slides. The resulting sample stack was then ultrasonically cored on the cross-section using a $2.3 \mathrm{~mm}$ cutter then glued, using high-strength epoxy, into a $3 \mathrm{~mm}$ brass tube for support. After mechanical thinning, polishing and dimpling, the samples had a final centre thickness $<10 \mu \mathrm{m}$. Samples were milled in a GATAN precision ion polishing system (PIPS) with 4 $\mathrm{keV} \mathrm{Ar}$ ions at $0.5 \mathrm{~mA}$ and an incident gun angle of $4^{\circ}$. Samples were milled until a perforation appeared at the $\mathrm{SiO}_{2}$ /glass interface. Samples were then examined with a Philips CM 300 microscope operating at $300 \mathrm{kV}$. SAXS samples were also prepared as described in Ref. [5]. SAXS measurements were performed at beamline 13 of the Australian Synchrotron.

Figure 1 shows XTEM micrographs of the embedded NPs before (a) and after (b) SHII with a fluence of $2 \times 10^{14} / \mathrm{cm}^{2}$. The as-formed spherical NPs have a broad Gaussian distribution, centered at approximately $14.5 \mathrm{~nm}$. The electron diffraction patterns (not shown) demonstrate that the well-defined and continuous rings resulting from randomlyoriented polycrystalline Ni NPs remain upon irradiation. The transformation from spherical to rod-like shape is readily apparent, as is the nanorod alignment with the incident ion beam direction. The minor dimension (width) of the SHII NPs and the unirradiated NPs determined from the SAXS analysis is shown in figure 2. The 
distribution for nanorod widths has clearly narrowed relative to the as-formed NPs and demonstrates the capabilities of SHII for the production of aligned nanorods of a specific width. We suggest that the elongation results from a combination of in-plane strain and NP melting generated by the energetic Au ions.

[1] A. Meldrum et al., Adv. Mater. 13, 1431 (2001).

[2] K. L. Kelly et al., J. Phys. Chem. B 107, 668 (2003).

[3] C. D'Orleans et al., Phys. Rev. B 67, 220101 (R) (2003).

[4] A. Oliver et al., Phys. Rev. B 74, 245425 (2006).

[5] R. Giulian et al., Phys. Rev. B 78, 125413 (2008).

[6] M. C. Ridgway et al., Nucl. Instrum. Methods Phys. Res. B 267, 931 (2008).
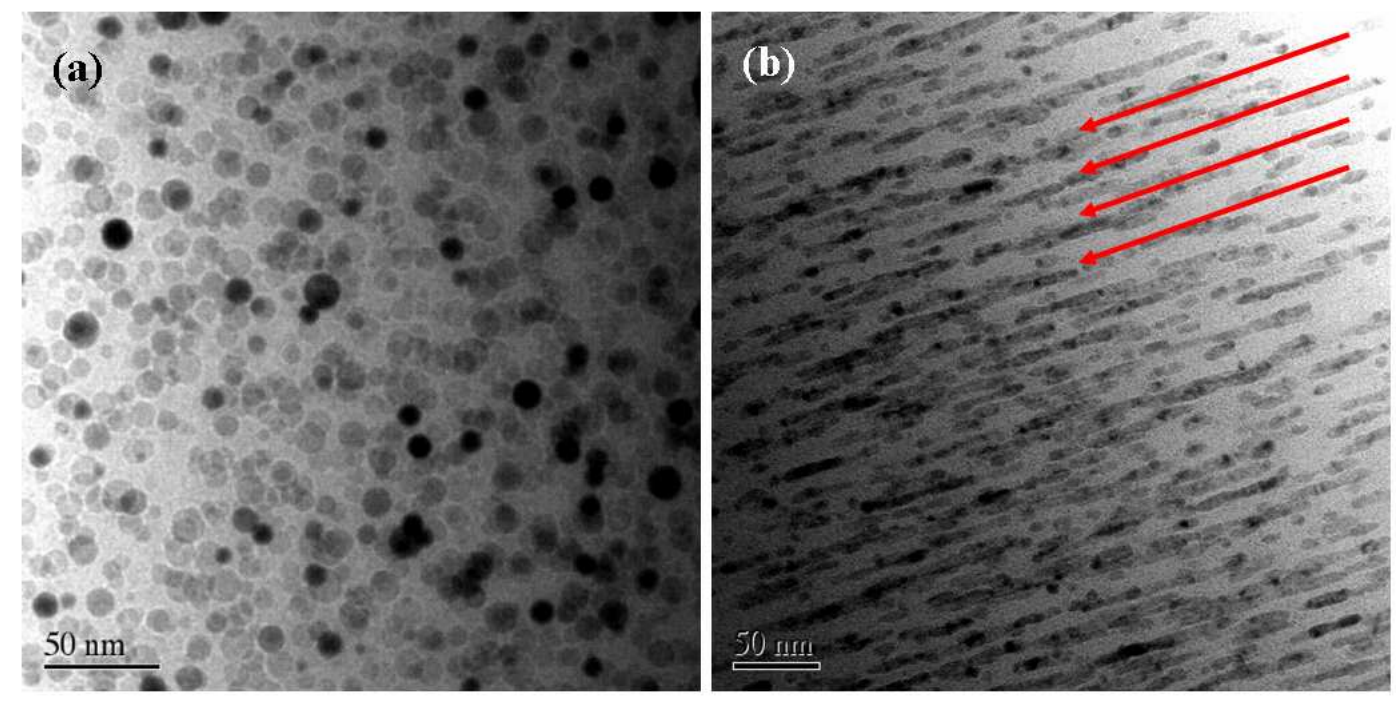

Figure 1. XTEM micrographs for (a) unirradiated and (b) $185 \mathrm{MeV}$ SHII NPs after a fluence of $2 \times 10^{14} / \mathrm{cm}^{2}$. The insets show electron diffraction patterns. The red arrows in (b) show the direction if the Au ion beam.

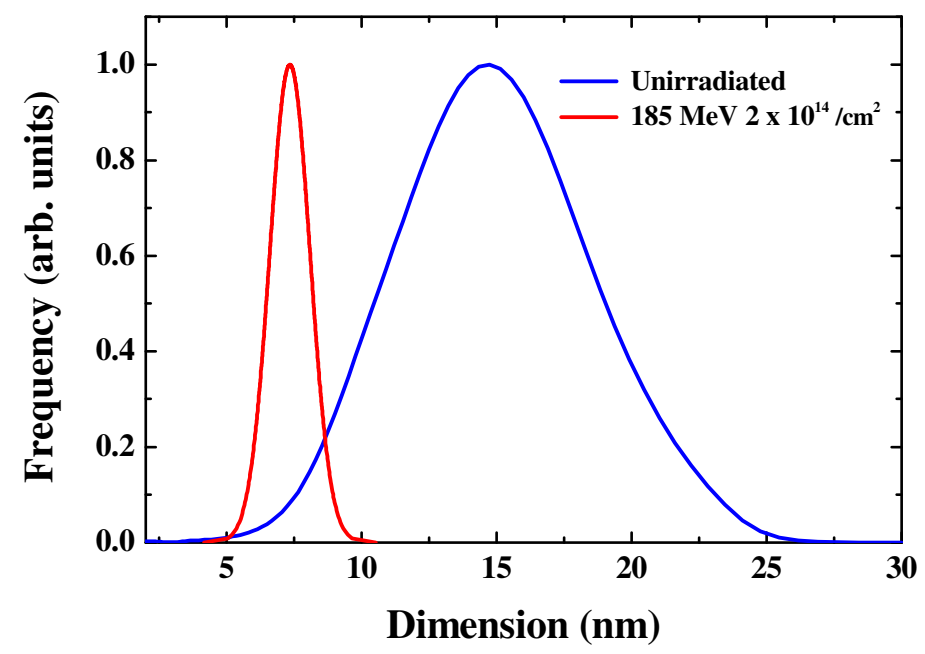

Figure 2. Minor dimension distributions determined from the SAXS analysis for the unirradiated (blue) and SHII NPs (red) given in figure 1. 